\title{
An easterly tip jet off Cape Farewell, Greenland. II: Simulations and dynamics
}

\author{
S. D. Outten,* I. A. Renfrew and G. N. Petersen \\ School of Environmental Sciences, University of East Anglia, UK
}

\begin{abstract}
An easterly tip jet that occurred on 21 February 2007 off Cape Farewell, Greenland, is examined. In Part I of this article aircraft observations were described. Now, in Part II, numerical simulations and an analysis of the dynamical forcing mechanisms are presented. The simulations make use of a limited-area $12 \mathrm{~km}$ resolution configuration of the Met Office's Unified Model. Sea-surface temperatures and sea-ice concentrations have been replaced using the Operational Sea Surface Temperature and Sea Ice Analysis (OSTIA) product, addressing a boundary-layer temperature bias, while roughness lengths over sea ice have been updated, addressing a wind-speed bias. These modifications ensured a reasonably accurate simulation: generally within $1-2 \mathrm{~K}$ and $2-3 \mathrm{~m} \mathrm{~s}^{-1}$ when compared with dropsonde observations. A momentumbudget analysis along a curved locus through the core of the jet has been derived. Off southeast Greenland, the easterly tip jet was in cross-jet geostrophic balance, but was being accelerated downstream by an along-jet pressure gradient. Over the curved part of the locus, as the jet rounded Cape Farewell, a cross-jet residual suggests that the jet was unbalanced at the height of the jet core. This residual decreases with height so that an approximate gradient wind balance applies in the upper part of the jet. The anticyclonic curvature, characteristic of easterly tip jets, was caused by a dramatic decrease in the cross-jet pressure-gradient force at the end of the barrier, after which the jet aligned with the synoptic-scale isobars and returned to approximate geostrophic balance. The momentum budget is shown to be robust and applicable to other cases. Copyright (C) 2009 Royal Meteorological Society
\end{abstract}

KEY WORDS Greenland Flow Distortion experiment; unified model; topographic flow; barrier wind

Received 15 January 2009; Revised 20 September 2009; Accepted 24 September 2009

\section{Introduction}

Greenland's size, extreme topography and location means that it has a significant impact on the weather and climate of Northern Europe. As the third largest mountain range in the Northern Hemisphere, it has an important influence on the local air flow and plays a major role in gravity-wave generation (Petersen et al., 2005), cyclogenesis (Kristjánsson and McInnes, 1999) and the formation of katabatic flows (Heinemann and Klein, 2002). Furthermore, interactions between Greenland and the cyclones passing close by on the North Atlantic storm track give rise to a number of topographically forced weather systems such as barrier flows and tip jets (Moore and Renfrew, 2005; Renfrew et al., 2008; Petersen et al., 2009; Renfrew et al., 2009a).

This article follows the observational analysis of the easterly tip jet (ETJ) event of 21 February 2007 presented in Renfrew et al. (2009a, hereafter Part I), where a more detailed background of ETJs is provided. These observations were based on the first flight (B268) of an aircraft-based field campaign: the Greenland Flow Distortion experiment (GFDex). An overview of the GFDex campaign is given in Renfrew et al. (2008). Flight

\footnotetext{
${ }^{*}$ Correspondence to: S. D. Outten, School of Environmental Sciences, University of East Anglia, Norwich, NR4 7TJ, UK.

E-mail: s.outten@uea.ac.uk
}

B268 consisted of three high-altitude legs across the jet at 20-25 thousand feet with four dropsondes released on each of these legs, three low-altitude legs flown at the aircraft's minimum safe altitude, approximately $35-50 \mathrm{~m}$ above the sea, and a profile descent and profile ascent before and after the low-level legs.

In this article a detailed analysis of a series of numerical modelling experiments is carried out. These make use of numerical weather prediction (NWP) simulations of the ETJ event using the Met Office's Unified Model. Further details of the model simulations are described in section 2. The numerical simulations are validated against the aircraft-based observations described in Part I, in particular the dropsonde observations. The simulations are also validated against radiosonde and satellite data in regions away from the ETJ. The simulations are discussed and validated in section 3. Diagnostics from the model are used to investigate the dynamics of the ETJ event, addressing the question: 'What is the balance of forces?' The climatological study of Moore and Renfrew (2005) gave insight into the occurrence of easterly tip jets and hypothesized a dynamical mechanism, suggesting that ETJs were the result of a geostrophically balanced barrier wind that moves to gradient-wind balance once it passes the end of the barrier. Recent studies of ETJs around Greenland have focused on the associated air-sea interaction, due to its importance in providing atmospheric forcing for convection in the ocean to the southwest of 


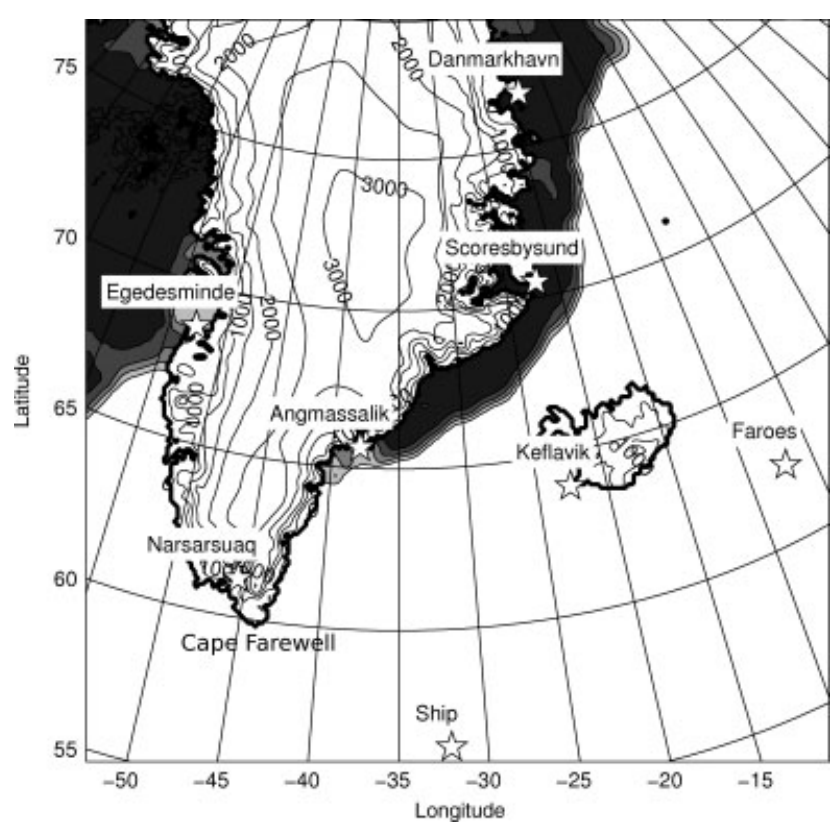

Figure 1. The full model domain, shown with the orography (contours every $500 \mathrm{~m}$ ) and the OSTIA sea-ice concentrations (shading every $20 \%$ ). The locations marked with stars are the launch locations for the radiosondes used in this work.

Cape Farewell (Martin and Moore, 2007; Petersen and Renfrew, 2009; Sproson et al., 2008). While dynamical mechanisms forcing westerly tip jets were documented by Doyle and Shapiro (1999) and further discussed by Petersen et al. (2003) and Moore and Renfrew (2005), no such investigation of the dynamics of ETJs has been published. Sections 4 and 5 describe this dynamical investigation, before three other cases are briefly discussed in section 6. Finally, the article is concluded in section 7 .

\section{Details of the numerical simulations}

Simulations of the ETJ examined in this article were carried out using the Met Office Unified Model (MetUM) version 6.1 in its limited-area configuration. This is a non-hydrostatic model, which uses a semi-implicit predictor-corrector scheme with semi-Lagrangian advection (Davies et al., 2005). The model employs a rotated hybrid-height, terrain-following grid, with both vertical and horizontal staggering. The MetUM also contains a full suite of physical parametrizations, including a Richardson-number-based boundary-layer scheme (Lock et al., 2000). For our simulations, a limited-area domain of $220 \times 220$ grid points was used, with a grid spacing of $0.11^{\circ}$ or approximately $12 \mathrm{~km}$. The vertical grid extends to approximately $40 \mathrm{~km}$ and consisted of 76 levels with a stretched grid to allow high resolution (26 levels) in the atmospheric boundary layer (ABL). The model was initialized from an operational Met Office global model run, which itself was initialized from a global analysis. The full limited-area domain is shown in Figure 1.

The Met Office's operational default sea-surface temperature (SST) and sea-ice concentration fields were replaced with data from the Operational Sea Surface
Temperature and Sea Ice Analysis (OSTIA). OSTIA uses satellite data from the Global High-Resolution Sea Surface Temperature (GHRSST) project (Donlon et al., 2007) and in situ observations from buoys to create a daily analysis of the SST with global coverage, at a resolution of approximately $0.05^{\circ}$. Further details of OSTIA can be found in Stark et al. (2007). The OSTIA analyses for both SST and sea ice are now used operationally by the UK Met Office, but were not at the time of GFDex. Early simulations of the ETJ used sea-ice concentrations from the Special Sensor Microwave/Imager (SSM/I) instrument (Wentz, 1997). While these were found to be very similar to the OSTIA sea-ice concentrations in most locations, there were notable differences in both the location and the concentrations of sea ice in the vicinity of Cape Farewell. In order that the sea ice should align better with the OSTIA SST field, the OSTIA seaice field was used. In the next section we will compare a default simulation (default-UM) with a simulation with the adjustments mentioned (OSTIA-UM) and show that the adjustments improve the model performance.

The MetUM uses three roughness lengths in parametrizing the air-sea turbulent momentum fluxes: the roughness length over open water, $Z_{0 \text { sea }}$, over sea ice, $Z_{0 \text { ice }}$, and over the marginal ice zone, $Z_{0 \text { MIZ. }}$ The operational default values of roughness lengths over sea ice and the marginal ice zone in the MetUM were found to be higher than recent research suggests, resulting in too much surface drag and consequently a reduced low-level wind speed. The default values were $Z_{0 \text { ice }}=$ $3.0 \times 10^{-3} \mathrm{~m}$ and $Z_{0 \mathrm{MIZ}}=1.0 \times 10^{-1} \mathrm{~m}$. These were replaced with values from the more recent research of Andreas et al. (2005), namely $Z_{0 \text { ice }}=5.0 \times 10^{-4} \mathrm{~m}$ and $Z_{0 \text { MIZ }}=5.0 \times 10^{-4} \mathrm{~m}$. These same values are used by the version of the MetUM used by the Hadley Centre (J. Edwards, personal communication).

The model was initialized at 0000 UTC on 21 February 2007, and run for $24 \mathrm{~h}$ using a $5 \mathrm{~min}$ time step. Figure 2 provides an overview of the main model simulation. The plots are not over the full domain, but rather a $100 \times 100$ grid point area centred on Cape Farewell. This provides a more detailed look at the ETJ. The $10 \mathrm{~m}$ wind speeds reach a maximum of around $30 \mathrm{~m} \mathrm{~s}^{-1}$, with anticyclonic curvature and a definite broadening once the jet has rounded the Cape. The strong shear zone at the northern edge of the ETJ (once it has passed the Cape) becomes stronger with height, and, when examined, was found to extend from the surface to well over a kilometre into the atmosphere (not shown). This strong shear zone is examined in more detail in Outten (2009). The two flows entering the eastern edge of the figure at around $65^{\circ} \mathrm{N}$ and $60^{\circ} \mathrm{N}$ are formed by a splitting around Iceland of the easterly flow, and can be seen to surround the more slowly moving air of the Iceland wake discussed in Part I. The easterly-to-southeasterly flow seen over the Greenland plateau is the southern edge of the air flow around the high-pressure centre located over central Greenland. Along the east coast of Greenland a tongue of cold air extends south, creating a strong horizontal temperature gradient perpendicular to the coast. The steep 
orography gives rise to a perturbation in the isobars, and creates a high-pressure ridge along the east coast, in agreement with theory (Smith, 1982). A strong horizontal pressure gradient perpendicular to the orography, as seen in this simulation, is often the driving force behind barrier flows (Schwerdtfeger, 1975; Parish, 1983).

The two parameters controlling how the oncoming air flow interacts with the orography of Greenland are the non-dimensional mountain height $(\hat{h}=N h / U)$ and the Rossby number $\left(R_{0}=U /(f L)\right)$, which were found (at 1300 UTC) to be approximately 2.5 and 0.42 respectively at an upstream location. These values are similar to those found by Petersen et al. (2003), and they suggest that
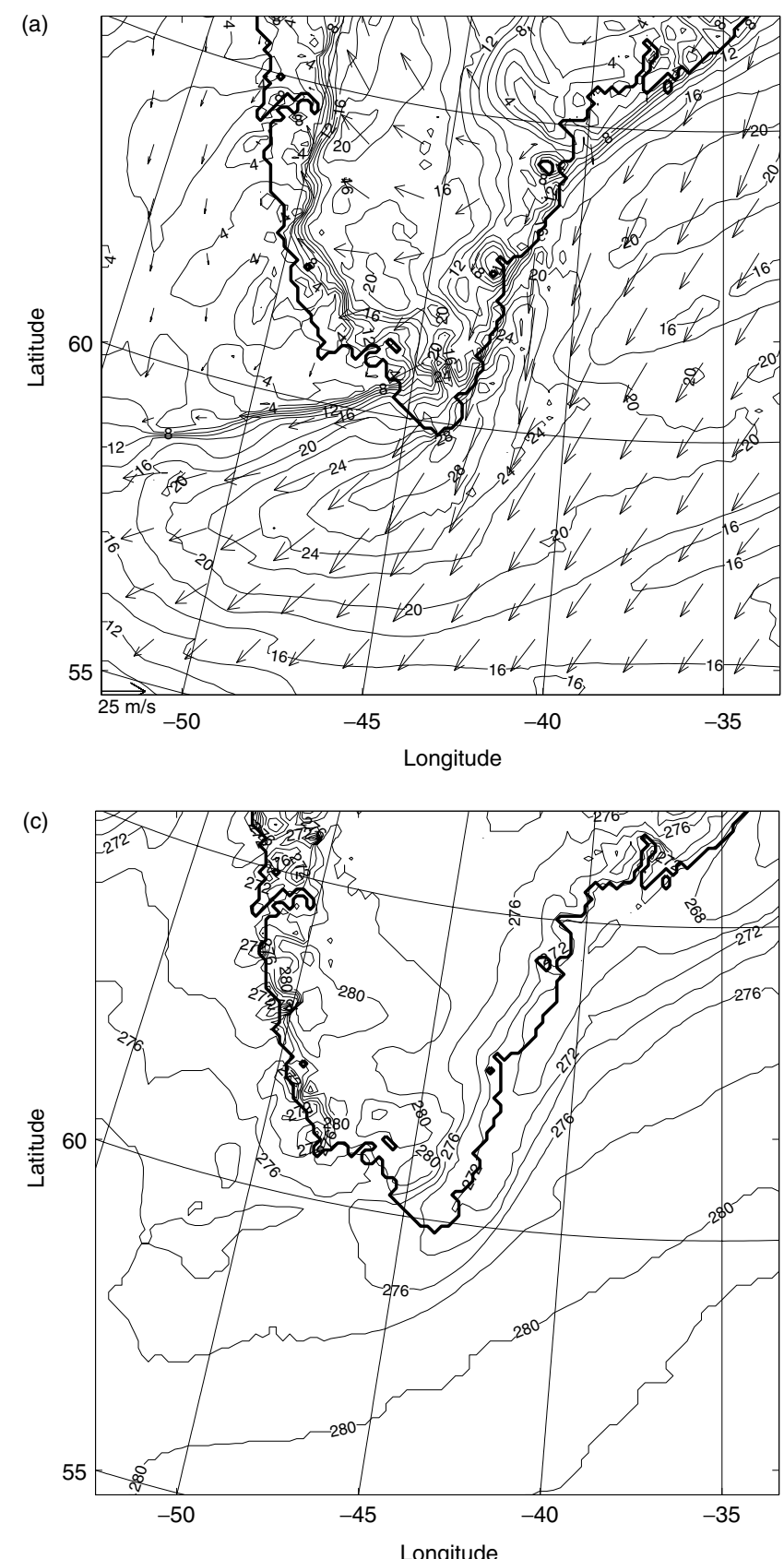

the flow will be blocked by the orography. Upstream, where the easterly flow first impinges upon the orography of Greenland, a region of flow splitting is formed to the north of the Denmark Strait (not shown). The part of the air flow that moves south through the Denmark Strait becomes slightly cross-isobaric. As this flow moves into the region of the ETJ shown in Figure 2 the air decelerates, which decreases the Coriolis force it experiences, and thus it begins to curve to the left (in the Northern Hemisphere) due to the background pressure gradient. This has the effect of making the flow more cross-isobaric as it approaches the high-pressure ridge along the east coast. A similar deceleration was
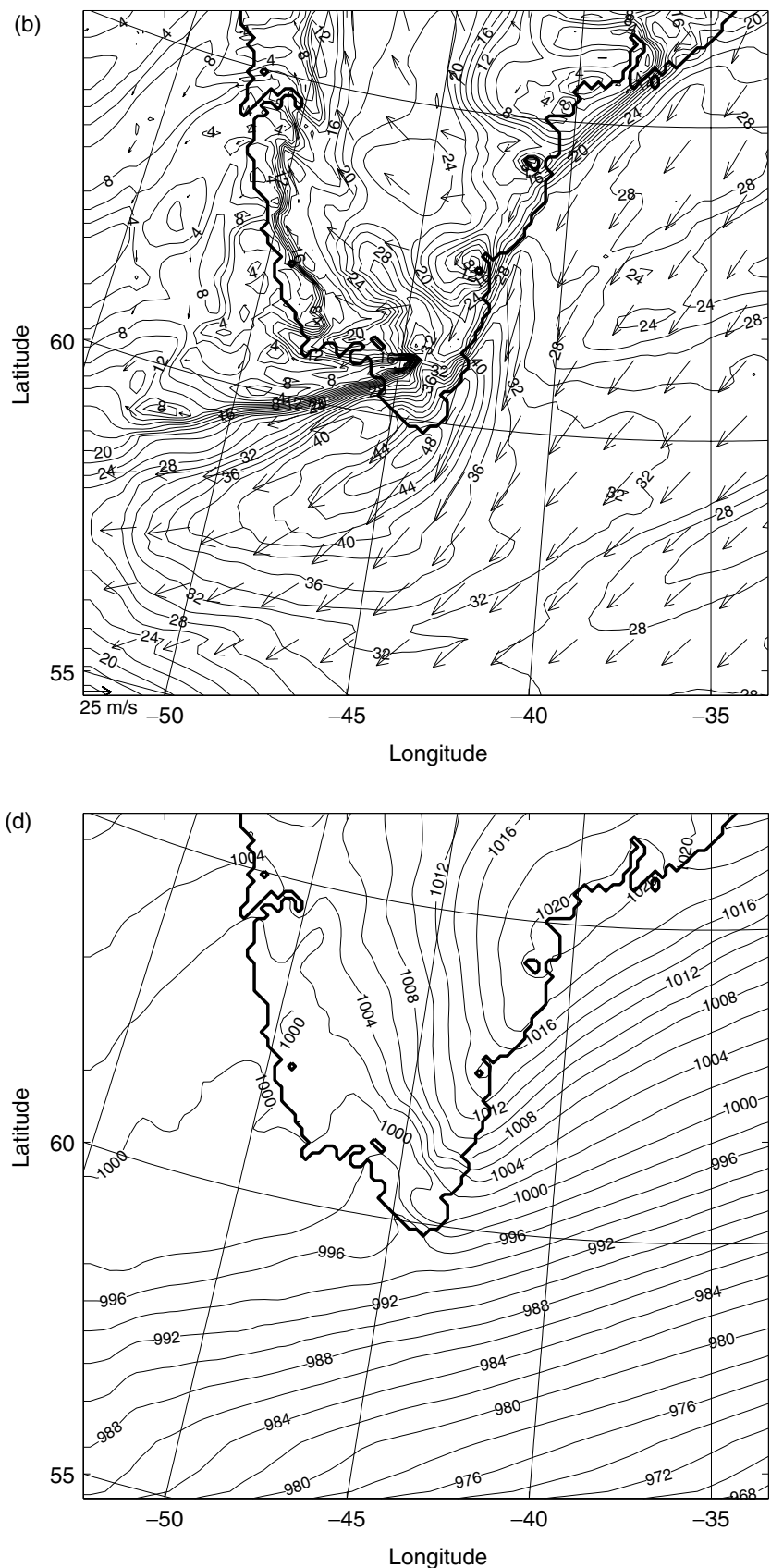

Figure 2. Numerical simulations at 1300 UTC on 21 February 2007 . The panels show (a) $10 \mathrm{~m}$ wind speed in $\mathrm{m} \mathrm{s}^{-1}$, (b) wind speed for mode level $12(660 \mathrm{~m})$ in $\mathrm{m} \mathrm{s}^{-1}$, (c) potential temperature in $\mathrm{K}$ at $5 \mathrm{~m}$ and (d) MSLP in hPa. Contour intervals are $2 \mathrm{~ms}$, $2 \mathrm{~m} \mathrm{~s}{ }^{-1}, 2 \mathrm{~K}$ and $2 \mathrm{hPa}$ respectively. Wind vectors are plotted at every eighth grid point and a unit vector is shown at the bottom left of each plot. 
observed by Barstad and Grønås in their investigation of 'left-sided jets' (Barstad and Grønås, 2005). The crossisobaric nature of the flow as it moves into the ETJ will be discussed further in section 4. After the ETJ has passed the Cape and moved downstream, the flow aligns with the isobars, once it is clear of the orographic effects.

Figure 2 provides an overview of the synoptic situation as simulated by the MetUM. In short, there is a lowpressure system to the southeast of the Cape (not shown), a mainly easterly flow impacting upon the orographic barrier with a northeasterly, cross-isobaric flow extending down the southeast coast from the Denmark Strait, a tongue of cold air coming down the coast from the north, large horizontal temperature and pressure gradients along the southeast coast of Greenland, a strong jet curving anticyclonically as it rounds Cape Farewell and a strong gradient in wind speed as the ETJ pushes up against the calm air in the Greenland wake. This synoptic overview is in good agreement with the ECMWF operation analysis illustrated in Figure 1 of Part I. The mean sea-level pressure (MSLP) and geopotential height at $500 \mathrm{hPa}$ from this simulation were quantitatively compared with the ECMWF operational analysis, and varied from the analysis by an average of around $1.5 \mathrm{hPa}$ in MSLP and $101 \mathrm{~m}$ in geopotential height.
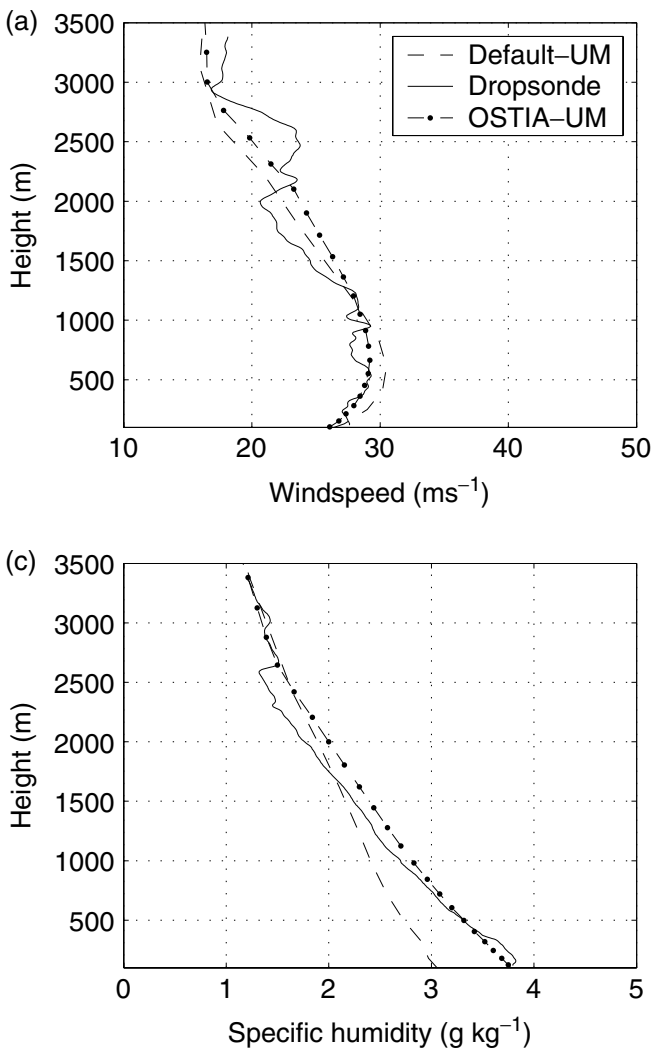

\section{Validation of the model simulations}

\subsection{Dropsonde comparisons}

In this section, profiles measured by the dropsondes released during flight B268 are compared with columns extracted from the model simulations at the nearest time step to the dropsonde's launch time, and at the nearest grid point to the dropsonde's launch location. Figure 3 shows the comparison for dropsonde 2, the second dropsonde on the 'northern leg'. The measurements from this dropsonde were used in both the 'northern' cross-section and the 'along-jet' cross-section in Part I (Figures 4-6 of that article), and are typical of the dropsondes from the along-jet leg. For ease of reference, the simulation with no modifications will be referred to as the default-UM, and the simulation with all adjustments included will be referred to as the OSTIA-UM. The location of the dropsonde and the $10 \mathrm{~m}$ wind speed are shown in the fourth panel.

The dropsonde shows a jet in the lowest $2000 \mathrm{~m}$, with a secondary maximum between $2000-3000 \mathrm{~m}$. While the model captures the overall shape of the profile, it does not distinguish between the main jet and the secondary maximum seen in the observations. The OSTIA-UM simulation shows an average variation from the measurements of less than $1 \mathrm{~K}$ in the potential temperature profile, and less than $0.15 \mathrm{~g} \mathrm{~kg}^{-1}$ in specific humidity profile. However the default-UM shows a boundary layer that is $4 \mathrm{~K}$

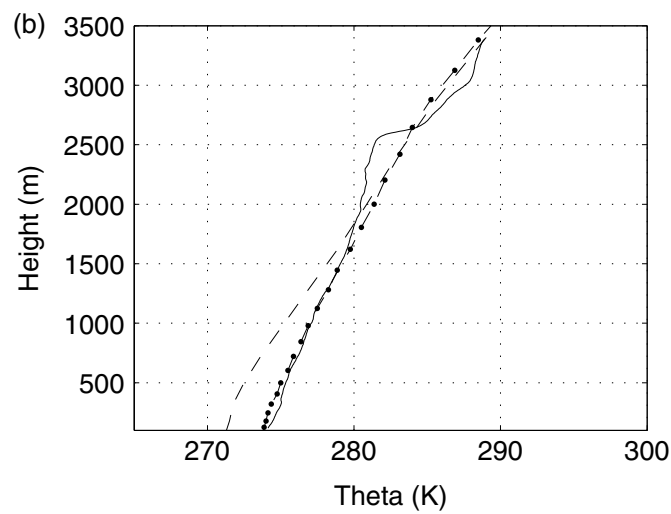

(d)

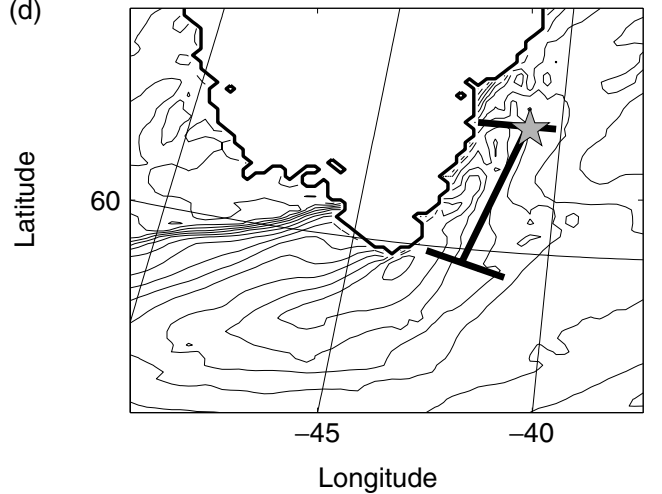

Figure 3. A comparison of the sounding from dropsonde 2 (solid line) with a column extracted from the MetUM with default settings (dashed), and with sea ice and SST from OSTIA and improved roughness lengths (dashed with markers). The panels show (a) wind speed in $\mathrm{ms}^{-1}$, (b) potential temperature in $\mathrm{K}$, (c) specific humidity in $\mathrm{g} \mathrm{kg}^{-1}$ and (d) an $x-y$ plot of $10 \mathrm{~m}$ wind speed over the sea with the locations of the dropsonde legs in bold and the location of dropsonde 2 marked by a grey star. 

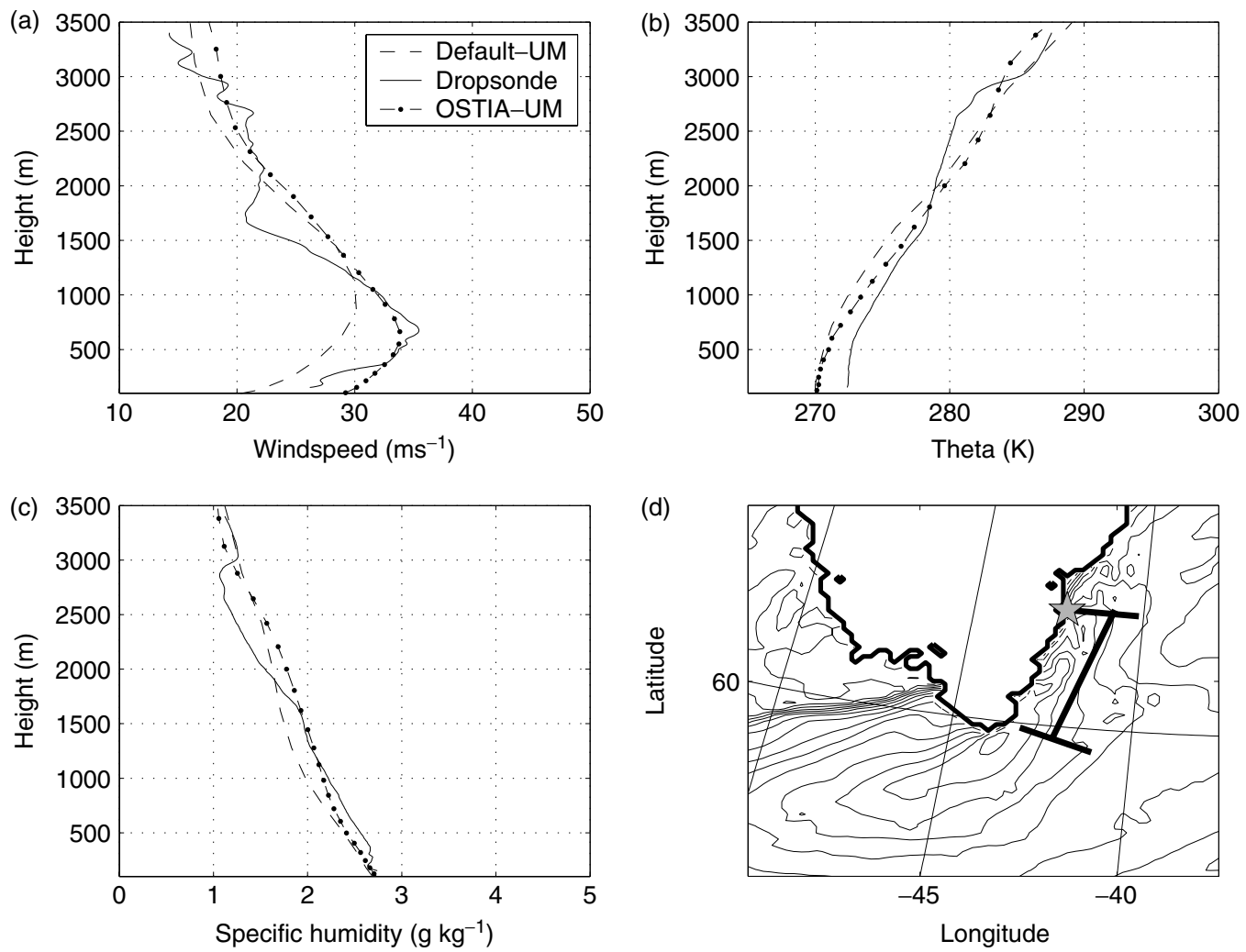

Figure 4. A comparison of the sounding from dropsonde 4 (solid line) with a column extracted from the MetUM with default settings (dashed), and with sea ice and SST from OSTIA and improved roughness lengths (dashed with markers). The panels show (a) wind speed in m $\mathrm{s}^{-1}$, (b) potential temperature in $\mathrm{K}$, (c) specific humidity in $\mathrm{g} \mathrm{kg}^{-1}$ and (d) an $x-y$ plot of $10 \mathrm{~m}$ wind speed over the sea with the locations of the dropsonde legs in bold and the location of dropsonde 4 marked by a grey star.

too cold and $0.8 \mathrm{~g} \mathrm{~kg}^{-1}$ too dry. These differences are similar to those seen in most of the other dropsondes. Dropsonde 2 was located at the northern end of the jet and slightly further offshore than the jet core. This explains the high wind speed but the lack of a sharply defined jet. The temperature inversion at around $2600 \mathrm{~m}$ in the dropsonde profile is not reproduced by the model. Petersen et al. (2009) observed similar inversions in their study of barrier flows, and suggested that this was because the initial conditions for the simulation do not contain this inversion. The relatively poor simulation of this temperature inversion is a common feature of the case study examined here, and may partly explain the model's lack of definition of the top of the jet in the wind-speed profiles.

In the northern leg, the closest dropsonde to the core of the jet was dropsonde 4, which was also the closest dropsonde to the coast. Figure 4 illustrates a welldefined jet peaking at around $35 \mathrm{~m} \mathrm{~s}^{-1}$, at a height of about $700 \mathrm{~m}$, in this dropsonde profile. As was seen in dropsonde 2, this region of high wind speed extends to approximately $1500 \mathrm{~m}$ above sea level, with a secondary maximum between here and approximately $3000 \mathrm{~m}$. The default-UM fails to capture the full magnitude of the jet, reaching a peak speed of only $30 \mathrm{~m} \mathrm{~s}^{-1}$ at a height of around $1000 \mathrm{~m}$. The inclusion of improved sea ice and roughness lengths in the OSTIA-UM simulation improves this, reproducing the observed peak wind speed to within $1 \mathrm{~m} \mathrm{~s}^{-1}$. According to both the OSTIA dataset (Figure 1) and SSM/I passive microwave observations, dropsonde 4 descended over sea ice, and so is over sea ice as far as the model is concerned. The jet is still vertically deeper in the simulations than was measured by the dropsondes, but overall the correspondence is very good. This comparison is of particular interest because of the potential temperature profile. It shows a boundary layer that is around $3 \mathrm{~K}$ too low in both simulations. The inclusion of the OSTIA SST field has not corrected this difference. However, the location of dropsonde 4 in the model is over sea ice in both simulations. If dropsonde 4 came down over open water or marginal ice, this would explain the warmer ABL that was observed.

The most well-defined wind-speed jet profile was seen in dropsonde 9, which was the closest dropsonde to the coast in the southern leg (Figure 5). It was the dropsonde closest to the core of the ETJ and measured the strongest wind speed of the flight, $49 \mathrm{~m} \mathrm{~s}^{-1}$. The jet was sharply defined between 200 and $1200 \mathrm{~m}$ above the surface, with the peak winds occurring at around $600 \mathrm{~m}$, and the jet top at $\sim 3000 \mathrm{~m}$. There was a strong shear at the top of the jet, and a very strong wind shear at the bottom of the jet. The model was unable to reproduce these strong wind shears and the very peak wind speeds in any of the simulations. The maximum simulated wind speed was around $45 \mathrm{~m} \mathrm{~s}^{-1}$. The average difference in wind speed between the default-UM and the OSTIA-UM profiles is less than $0.5 \mathrm{~m} \mathrm{~s}^{-1}$ at this location. While the OSTIA-UM did simulate the observations of potential temperature and 

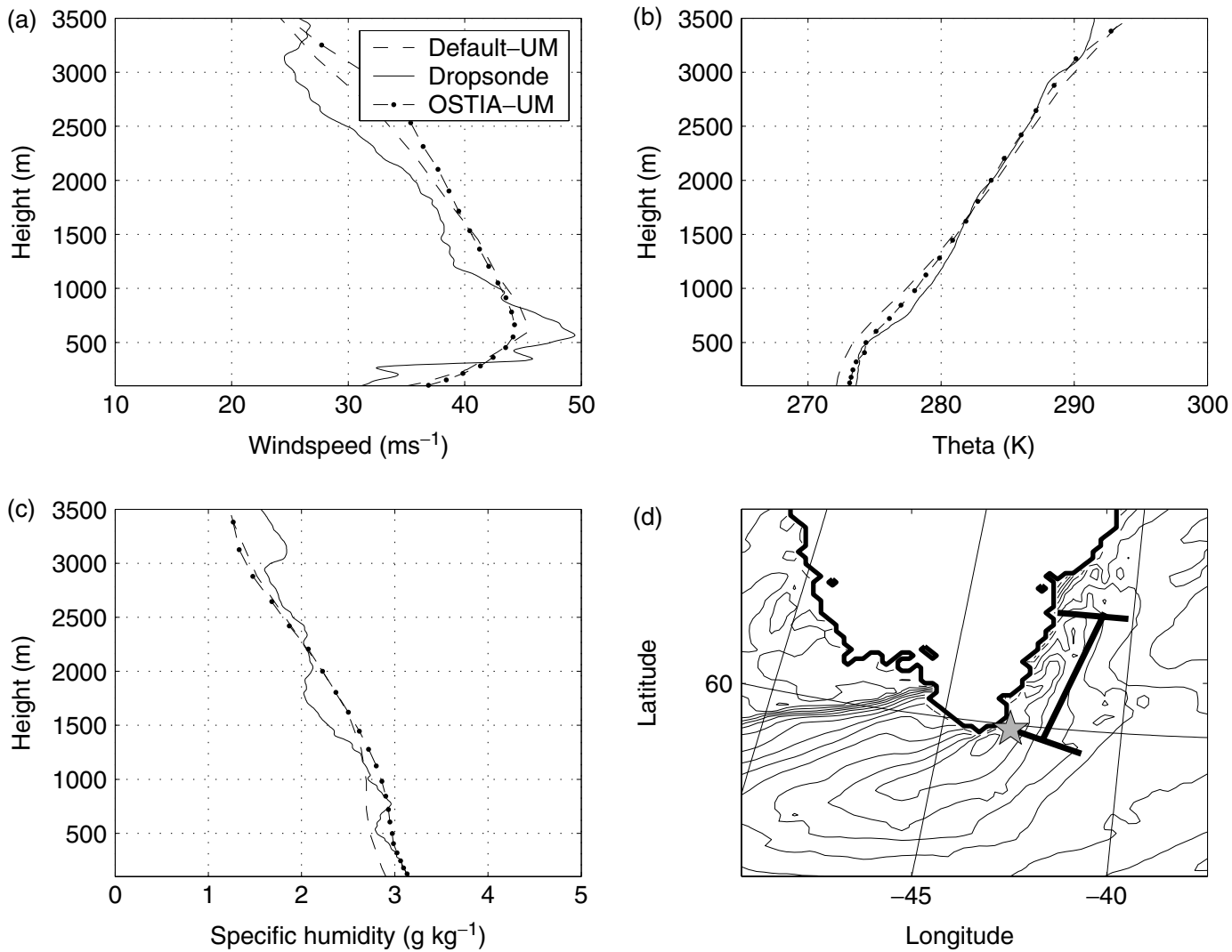

Figure 5. A comparison of the sounding from dropsonde 9 (solid line) with a column extracted from the MetUM with default settings (dashed), and with sea ice and SST from OSTIA and improved roughness lengths (dashed with markers). The panels show (a) wind speed in $\mathrm{ms}^{-1}$, (b) potential temperature in $\mathrm{K}$, (c) specific humidity in $\mathrm{g} \mathrm{kg}^{-1}$ and (d) an $x-y$ plot of $10 \mathrm{~m}$ wind speed over the sea, with the locations of the dropsonde legs in bold and the location of dropsonde 9 marked by a grey star.

specific humidity in the boundary layer more accurately, the differences from the default-UM simulation are only around $1 \mathrm{~K}$ and $0.2 \mathrm{~g} \mathrm{~kg}^{-1}$ at the surface, much smaller than was seen in dropsonde 2 and in many of the other dropsondes.

In summary, a reasonable simulation of this ETJ event was obtained with the MetUM after several modifications to the MetUM's default settings were made.

- Roughness lengths of sea ice and the marginal ice zone were modified, based on more recent observations, which improved the simulated wind profiles over sea ice.

- The SST and sea-ice ancillary files were modified, making use of new high-resolution fields, which eliminated a cold and dry bias in the ABL of up to $4 \mathrm{~K}$ and $1 \mathrm{~g} \mathrm{~kg}^{-1}$.

Examining all of the comparisons when these adjustments have been applied to the model, some general observations can be made. The jet in the model is vertically deeper and smoother than observed. This may be partly due to the model's relatively poor simulation of a well-defined temperature inversion at the top of the ABL (2000-3000 m). The smoothness of the jet in the simulations is not the result of inadequate model resolution. At an early stage in this research, the model set-up was changed from using 38 model levels to the 76 vertical levels used here. This change resulted in only minor improvements in resolving the vertical structure and did not have any significant effect on the overall shape and smoothness of the jet in the model simulation. Above the jet there was a region of weaker wind speeds measured by all the dropsondes. In dropsonde profiles where the jet reaches maximum wind speeds of around $30 \mathrm{~m} \mathrm{~s}^{-1}$, the wind speed is almost constant through the jet profile. This is clearly visible in the along-jet dropsondes shown in Figure 6 of Part I. The wind speed in these profiles is accurately reproduced by the model. In dropsonde profiles where the wind speed exceeds $30 \mathrm{~m} \mathrm{~s}^{-1}$, the jet has a pronounced maximum with a strong wind shear above and below the jet. This was shown in dropsondes 4 and 9 (Figures 4 and 5), and also seen in dropsondes 10 and 11 (not shown). The model does not fully reproduce the highest wind speeds or the strong shears observed in these profiles. Dropsondes 4 and 9 were at the western end of the two cross-jet legs, and so were closest to the orography and to the core of the jet.

In the southern dropsonde leg, as the jet approaches the Cape, the peak winds increase, while remaining at approximately the same height. It also becomes warmer and more moist, which is consistent with the dropsonde measurements and the observations of a cold tongue of air coming down the coast (see Figures 5 and 6 of Part I).

The model was often seen to have a wind directional deviation of $5^{\circ}-15^{\circ}$ from the dropsonde wind direction (not shown), and no explanation for this has been found. 
This discrepancy is probably not significant and it has not been investigated further. Despite some differences, after the inclusion of the improved SST and sea-ice fields and the alterations to the roughness lengths over sea ice, the model simulates the ETJ event from the field campaign with reasonable accuracy. Similar comparisons were made to the measurements recorded by the flightlevel instruments during the low-level legs of the flight. These comparisons are covered in more detail in Outten (2009). Again, the changes to the model set-up resulted in improvements in these comparisons.

\subsection{Validation away from the jet}

On the day of the ETJ event, radiosondes were launched from nine locations within the model domain. Each location launched one radiosonde at 1100 UTC and another at 2300 UTC. The launch locations were Ammasalik (Angmassalik), Danmarkshavn, Aasiaat (Egedesminde), Narsarsuaq and Ittoqqortoormiit (Scoresbysund) in Greenland, Keflavik in Iceland, the Faroe Islands and two ships located at $56.48^{\circ} \mathrm{N}, 32.06^{\circ} \mathrm{W}$ and $56.37^{\circ} \mathrm{N}, 29.94^{\circ} \mathrm{W}$ at the time of the morning launch. These locations are marked in Figure 1 by stars. These are well dispersed across the model domain, with Danmarkshavn in the north, the Faroes close to the eastern edge of the domain, Egedesminde on the western coast of Greenland and the ships passing to the south of Greenland. Comparisons of model profiles with these radiosonde profiles were carried out as for the dropsondes (not shown). These comparisons showed that the model accurately reproduced the basic shape of the observed soundings. There were average differences of around $2-3 \mathrm{~m} \mathrm{~s}^{-1}$ in wind speed, around $1 \mathrm{~K}$ in potential temperature and around $0.1-0.2 \mathrm{~g} \mathrm{~kg}^{-1}$ in specific humidity. In general, the radiosonde comparisons provide further evidence of a good numerical simulation of this case.

As discussed in Part I, QuikSCAT is a space-based scatterometer that provides an estimate of the $10 \mathrm{~m}$ wind speed, based on the roughness of the sea surface. QuikSCAT winds retrieved using geophysical models from both NASA and Remote Sensing Systems (RSS) are illustrated in Figure 12 of Part I. The morning passes are shown again here, compared with the $10 \mathrm{~m}$ wind field from the model simulation (Figure 6). The QuikSCAT data are from two passes of the satellite. Since these passes occurred between approximately 0700 and 0900 UTC, the output from the model run for this comparison was taken at 0800 UTC. The QuikSCAT data are at a resolution of $25 \mathrm{~km}$, while the model data are at the model resolution of $12 \mathrm{~km}$. The model reproduces the broad structure seen with both retrievals of QuikSCAT. The model shows a maximum wind speed of $30 \mathrm{~m} \mathrm{~s}^{-1}$ both alongside the barrier and around the Cape. This compares well with the NASA retrieval, which shows the wind speeds in the core reaching $33 \mathrm{~m} \mathrm{~s}^{-1}$. The RSS retrievals show much larger wind speeds, reaching $39 \mathrm{~m} \mathrm{~s}^{-1}$ in the core, with much of the area around the Cape over $33 \mathrm{~ms}^{-1}$. The shape of the jet is slightly different between the three plots, extending
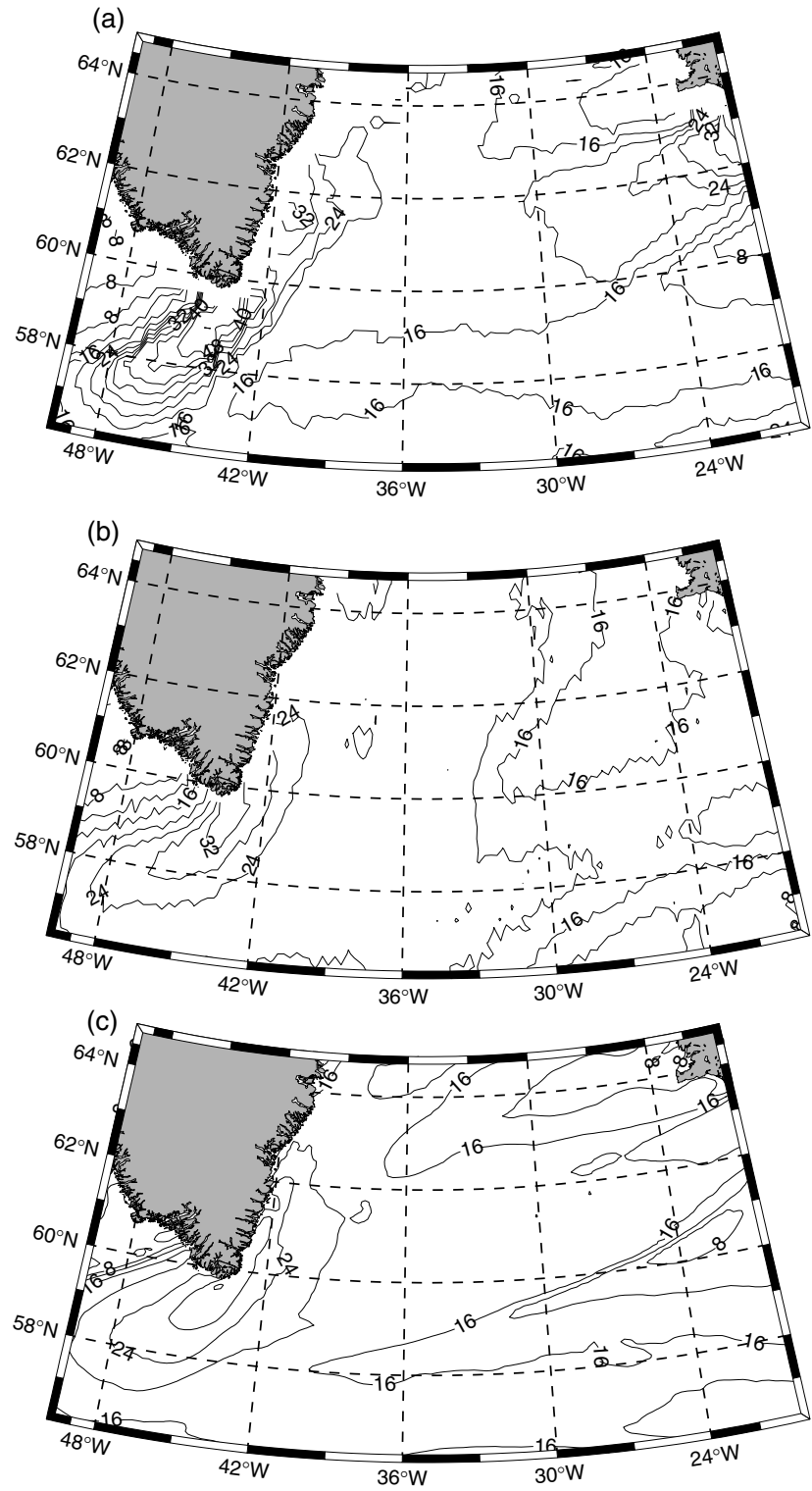

Figure 6. Estimated $10 \mathrm{~m}$ wind speed at 0800 UTC on 21 February 2007 from the RSS QuikSCAT retrieval (top), NASA QuikSCAT retrieval (middle) and the model simulations (bottom). Contours are shown for every $4 \mathrm{~m} \mathrm{~s}^{-1}$.

further north along the east coast of Greenland in the RSS retrieval and the simulation than it does in the NASA retrieval. In the simulation, the northern edge of the jet west of the Cape shows a steep gradient between the core wind speeds and the wake in the lee of Greenland. Both QuikSCAT retrievals show more gentle gradients in this region. There also appears to be more horizontal spreading of the jet in the simulation than in the satellite wind retrievals. This could suggest a failing in the simulation to capture correctly how the jet diffuses once it is clear of the orography, and how this diffusing jet interacts with the calm wake of Greenland. However, there were no measurements taken in the region of the shear zone during the field campaign, and an investigation of this phenomenon is beyond the scope of this article. It is covered in more detail in Outten (2009). 
In short, the QuikSCAT estimates of $10 \mathrm{~m}$ wind speed compare reasonably well spatially with the model simulations; however, there is a systematic difference, especially between the RSS retrieval and the model wind speeds in the jet. Several recent studies have noted the problems that such scatterometers have at retrieving accurate wind speeds for winds over about $20 \mathrm{~m} \mathrm{~s}^{-1}$ (Chelton and Freilich, 2005). Recent QuikSCAT validation studies in this area, using buoy observations (Moore et al., 2008) and a compilation of the GFDex aircraft observations (Renfrew et al., 2009b) illustrate a bias at higher wind speeds; QuikSCAT winds overestimate the observed winds for both the RSS and NASA retrievals. This bias may partly explain the differences seen here.

The distribution of cloud in the simulation was also examined by comparing the simulated liquid water content with images from the Advanced Very High Resolution Radiometer (AVHRR) in the visible and infrared bands (Figures 2 and 3 of Part I). This comparison showed many features that were qualitatively well simulated: curving clouds on the northern edge of the passing low-pressure system; a band of cloud extending from Iceland towards Cape Farewell and another extending north over Greenland; a small clear region in Iceland's wake and in Greenland's wake (not shown). Vertical profiles of liquid water content from the model at the locations of the profile ascent and descent from the flight were also examined (not shown). These show cloud layers at around 1000 and $2700 \mathrm{~m}$, and of similar depth to the aircraft measurements (Figure 7 of Part I).

\section{A dynamical analysis}

\subsection{Methodology}

With the validity of the model simulation established, diagnostics will now be used to investigate the balance of forces that control the jet. It will first be established that an Eulerian approach is appropriate. To do this, the variation of wind speed, potential temperature, and specific humidity was examined over a $24 \mathrm{~h}$ period at four approximately evenly spaced points, chosen to be in different dynamical regions of the jet. Figure 7 illustrates this variation for wind speed at the four points with point 1 being the northernmost point. The locations of these four points in the jet core are shown in Figure 8 by the grey squares. Assuming only horizontal motion and steady conditions, it was calculated that it would take approximately $3.8 \mathrm{~h}$ for an air parcel to travel the length of the jet. This time interval is shown in Figure 7 by the two vertical lines, indicating an air parcel entering the northern end of the jet at 1200 UTC and exiting the southwestern end $3 \mathrm{~h} 40 \mathrm{~min}$ later.

An inspection of Figure 7 reveals that over this period the wind speed varies by typically $\pm 2 \mathrm{~m} \mathrm{~s}^{-1}$. At point 3 the variation is a little higher at $\pm 5 \mathrm{~m} \mathrm{~s}^{-1}$. These changes are relatively small compared with the mean values of approximately $40 \mathrm{~m} \mathrm{~s}^{-1}$. The potential temperature and relative humidity were also examined, and found to vary

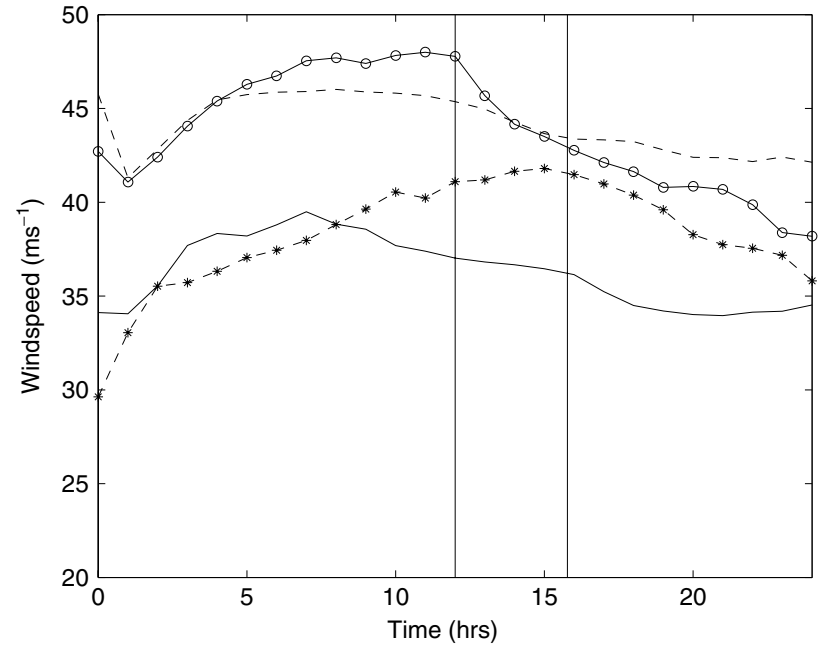

Figure 7. The variation in wind speed at point 1 (solid line), point 2 (dashed line), point 3 (circles) and point 4 (asterisks) along the jet, during 21 Februrary 2007, starting from 0000 UTC. The two vertical lines indicate the time taken for an air parcel to travel the length of the jet.

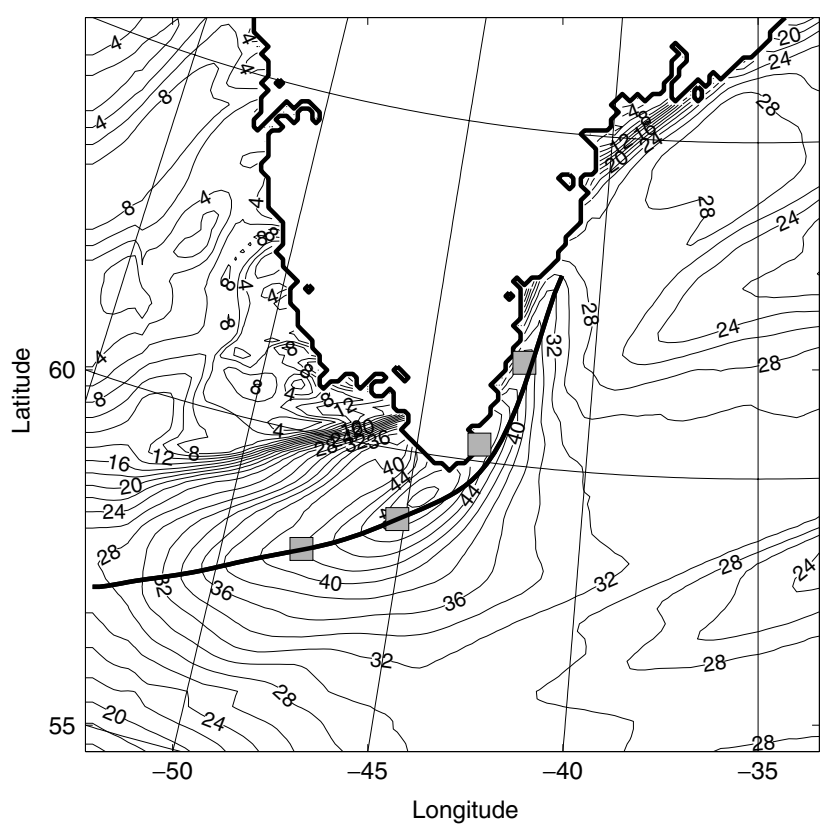

Figure 8 . Wind speed with contours every $2 \mathrm{~m} \mathrm{~s}^{-1}$ for model level 12 at 1300 UTC on 21 Februrary 2007. The jet locus used in the analysis is marked by the thick line. The four points used in confirming the validity of an Eulerian approach are shown as grey squares.

by approximately $1 \mathrm{~K}$ (with a daily mean of $275 \mathrm{~K}$ ) and $2-3 \%$ (with a daily mean of $91 \%$ ) at all four points. This suggests that over the time-scale of an air parcel travelling along the jet, the situation is approximately steady-state. For the remainder of this analysis all diagnostics taken from the model will be three-hour means centred around 1300 UTC (unless specifically stated otherwise). Using three-hour means ensures that the effect of unrepresentative instantaneous anomalies is minimized, and that minor terms can be neglected.

Diagnostics are extracted on the model's $x-y$ grid at model level 12 (660 m above the surface). This model 
level was selected because it is where the jet's maximum wind speed most often occurs. This is based on examining the model level at which the maximum wind speed occurs for each grid point in the jet, where the jet is defined as those grid points where $10 \mathrm{~m}$ wind speed $>25 \mathrm{~m} \mathrm{~s}^{-1}$. Although the model uses terrain-following vertical model levels, the height of model level 12 is the same at all points along the jet, since the core of the jet is over the sea.

To calculate a momentum budget, it is necessary to define a locus for the jet, i.e. a stroke curve through the higher wind speeds. To do this, the wind speeds on model level 12 were examined, and the grid point in each north-south row containing the maximum wind speed was found. This produced a single set of grid points running through the jet core along the length of the jet. A smooth ninth-order polynomial curve was fitted through these points using the method of least-squares. This curve, or jet locus, is shown by the solid line in Figure 8. A high-order polynomial curve was used for this purpose so as to ensure that the curve accurately captured the tight curvature of the jet close to Cape Farewell. Model diagnostics were interpolated on to this jet locus using a two-dimensional linear interpolation scheme. In short, the individual terms of the equations of motion were calculated on a curve through the jet core, at the height of maximum wind speed, using diagnostics averaged over three hours. Note this jet locus roughly follows a horizontal streamline, as elaborated on later.

\subsection{Momentum budget}

To carry out the momentum budget analysis, a commonly used approximation to the primitive equations was used, based on the framework of Stull (1988), which neglects minor terms, as appropriate when mean values are used. The momentum equations were separated into zonal (Eq. (1)) and meridional (Eq. (2)) components:

$$
\begin{aligned}
& \underbrace{\frac{\partial \mathrm{u}}{\partial \mathrm{t}}}_{\text {time derivative }}=-\underbrace{\bar{u} \frac{\partial \bar{u}}{\partial x}-\bar{v} \frac{\partial \bar{u}}{\partial y}-\bar{w} \frac{\partial \bar{u}}{\partial z}}_{\text {advection }}+\underbrace{f \bar{v}}_{\text {Coriolis }} \\
& -\underbrace{\frac{1}{\bar{\rho} \frac{\partial \bar{p}}{\partial x}}}_{\text {pressure }}+\underbrace{v\left[\frac{\partial^{2} \bar{u}}{\partial x^{2}}+\frac{\partial^{2} \bar{u}}{\partial y^{2}}+\frac{\partial^{2} \bar{u}}{\partial z^{2}}\right]}_{\text {viscosity }}-\underbrace{\frac{1}{\bar{\rho}\left[\frac{\partial\left(\overline{\rho u^{\prime} w^{\prime}}\right)}{\partial z}\right]},}_{\text {flux divergence }} \\
& \underbrace{\frac{\partial v}{\partial \mathrm{t}}}_{\text {time derivative }}=-\underbrace{\bar{u} \frac{\partial \bar{v}}{\partial x}-\bar{v} \frac{\partial \bar{v}}{\partial y}-\bar{w} \frac{\partial \bar{v}}{\partial z}}_{\text {advection }}-\underbrace{f \bar{u}}_{\text {Coriolis }} \\
& -\underbrace{\frac{1}{\bar{\rho}} \frac{\partial \bar{p}}{\partial y}}_{\text {pressure }}+\underbrace{v\left[\frac{\partial^{2} \bar{v}}{\partial x^{2}}+\frac{\partial^{2} \bar{v}}{\partial y^{2}}+\frac{\partial^{2} \bar{v}}{\partial z^{2}}\right]}_{\text {viscosity }}-\underbrace{\frac{1}{\bar{\rho}\left[\frac{\partial\left(\overline{\rho v^{\prime} w^{\prime}}\right)}{\partial z}\right]}}_{\text {flux_divergence }} .
\end{aligned}
$$

Note that a bar above any of the terms (e.g. $\bar{u}$ ) indicates that a three-hour mean value is used in the analysis. These equations are essentially the same as those

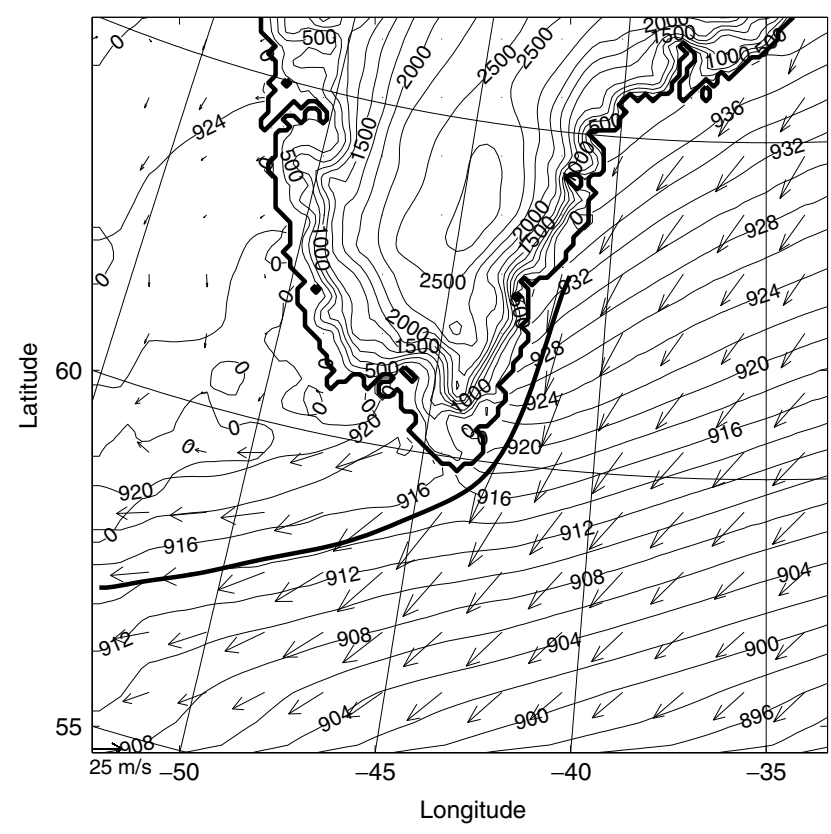

Figure 9. The pressure over the sea for model level 12, at $660 \mathrm{~m}$, with contours every $2 \mathrm{hPa}$. The wind vectors from the same level and the jet locus are also shown.

used in the MetUM version 5.5 and higher as documented by Staniforth et al. (2003); however, they are not identical, as the MetUM includes diffusion terms etc. In the model, the equations are used in the rotated latitude/longitude terrain-following coordinate system. In order to calculate the first- and second-order differentials at each point along the curve, diagnostics from the model were extracted over $3 \times 3 \times 3$ grid points, centred around each point of the jet locus. This allowed a centred-space, finite-difference approximation to be used to find these differentials.

It is important to note that since the terms have been calculated at points along the curve, the orientation of the jet relative to the $x$ and $y$ axes is different at each point. To account for this, at each point along the jet locus the terms in the momentum budget were calculated and a rotation matrix was applied that rotated the terms by the angle $\phi$, where $\phi$ was the angle between the wind direction and the $x$ axis. This method resulted in an along-jet, $s$, and cross-jet (or normal), $n$, component being obtained for each term of the momentum budget, at each point along the jet locus. In effect, the terms are calculated along a pseudo-streamline, since they are aligned with the wind direction, and the cross-jet component of the wind speed, $u_{n}$, is zero at all points along the locus.

Figure 10 shows the magnitude of the terms as a function of the distance along the jet locus, from the northern end of the jet at $0 \mathrm{~km}$ to the southwestern end at $850 \mathrm{~km}$. The jet locus passes directly south of Cape Farewell at approximately $380 \mathrm{~km}$ (cf. Figure 8 ). The four points previously examined to establish the validity of an Eulerian approach (Figure 8) are located at approximately $100,300,480$ and $630 \mathrm{~km}$. The five terms on the righthand side of the horizontal momentum equation (Eqs (2) and (1), top and bottom panel respectively) are plotted. 
The residual of these five terms combined is also plotted, and note this includes the time-derivative term from the left-hand side of Eqs (1) and (2). The magnitudes of these terms vary from 0 to $\pm 7 \times 10^{-3} \mathrm{~m} \mathrm{~s}^{-2}$. This is comparable to values found by Renfrew (2004) and Zhong and Whiteman (2008), who both performed a similar type of analysis for downslope flows.

The Coriolis force is zero at all points in the alongjet direction, which indicates that the rotation of the terms has correctly aligned the along-jet components with the wind direction, a consequence of the crossjet wind component, $u_{n}$, being zero (Figure 10). This means that the cross-jet component of Coriolis force is the total Coriolis force experienced by air parcels travelling through the jet. The plot of along-jet momentum budget also indicates that beyond $500 \mathrm{~km}$ the jet is approximately aligned with the local isobars, since the along-jet pressure gradient is approximately zero. The cross-jet Coriolis force is negative over the length of the jet, consistent with a Coriolis forcing into the barrier, i.e. perpendicular and to the right of the flow in the Northern Hemisphere. The Coriolis term smoothly increases in magnitude to its peak shortly after passing Cape Farewell, before decreasing as the jet moves downstream. This variation in the Coriolis term is almost entirely due to the variation in the along-jet wind speed, since the variation in the Coriolis parameter due to the change in latitude only accounts for $4 \times 10^{-6} \mathrm{~m} \mathrm{~s}^{-2}$ of the change in the force.

For the first $150 \mathrm{~km}$ of the jet, the cross-jet residual is approximately zero, and the pressure-gradient force
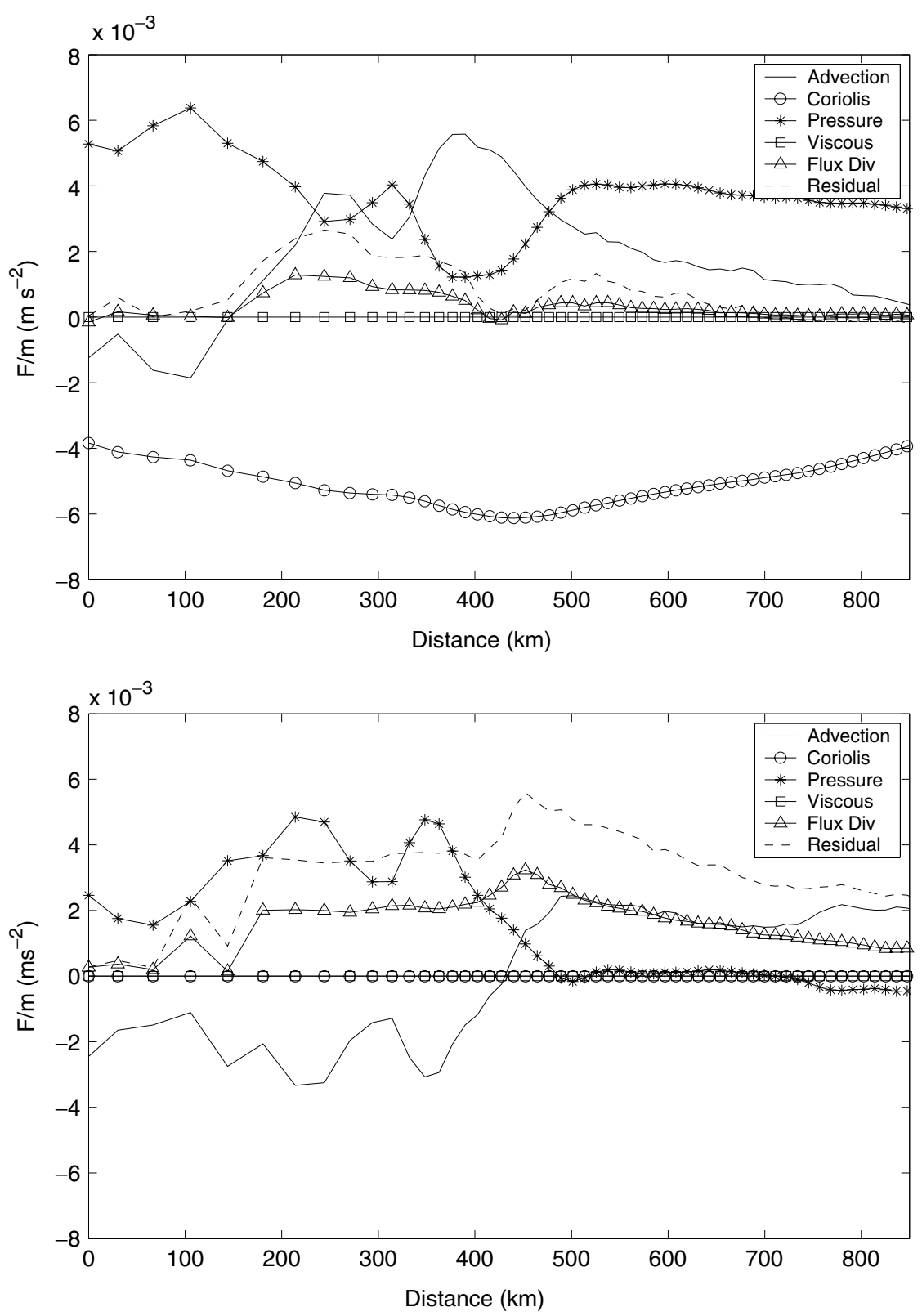

Figure 10. The cross-jet (top) and along-jet (bottom) components of the momentum budget, in terms of force per unit mass ( $F / m$ ), shown against distance along the jet locus. The forces shown are the advection (solid line), Coriolis (circles), pressure gradient (asterisks), viscous stress (squares) and turbulent momentum flux divergence (triangles). The residual is also shown (dashed line). 
is approximately balanced by the Coriolis force. This would suggest cross-jet geostrophic balance, and would be consistent with the presence of a barrier wind (Schwerdtfeger, 1975). However, it is important to note that the flow at this point in the jet is cross-isobaric in nature (Figure 9). This means that although the total Coriolis force experienced by an air parcel in this region is balanced by the normal component of the pressuregradient force, there is also an along-jet component of the pressure-gradient force that is not balanced by the Coriolis force, and this gives rise to an along-jet acceleration. The resulting increase in wind speed is illustrated via the cross-jet Coriolis force, which indicates that the jet is accelerating until around $450 \mathrm{~km}$.

Beyond $100 \mathrm{~km}$, the orography perpendicular to the jet locus decreases in height as the jet approaches the Cape. This causes the normal component of the pressuregradient force to decrease (Figure 10, top panel). Due to the along-jet component of the pressure-gradient force accelerating the jet, the wind speed remains high, as does the Coriolis force. Hence the Coriolis force becomes larger than the normal component of the pressure-gradient force, leading to the jet turning to the right of the direction of motion. Hence, as the orography decreases in height near Cape Farewell, an anticyclonic curvature is introduced into the jet. This curvature reaches a maximum at around $380 \mathrm{~km}$, as the jet rounds the Cape, and as the normal pressure gradient reaches its minimum. The decrease in cross-jet pressure-gradient force between 100 and $400 \mathrm{~km}$ is accompanied by an increase in the advection term. The distribution of the advection term along the jet is approximately inversely correlated with the distribution of the pressure-gradient force.

As the synoptic situation changes, so too does the pressure-gradient force. The jet reacts to these changes with variations in the advection term, since this has the fastest response, i.e. it can change rapidly in order to accommodate the changes occurring in the pressuregradient term. In this region, between approximately 150 and $450 \mathrm{~km}$, the jet is unbalanced, as indicated by the large residual. Approximately half of this residual comes from the increase in turbulent mixing as the jet curves around the Cape. Empirical evidence suggests that as the wind speed in a direction increases, the turbulence in the wind in that direction also increases (Stull, 1988). One interesting feature of the pressuregradient force in this region is the notable fluctuation that occurs between 230 and $330 \mathrm{~km}$. An investigation revealed that this fluctuation is due to the presence of the Danells and Lindenows Fjords. Their effect can be seen in Figure 9, which illustrates the region of the fluctuation by a spreading of the 920 and $922 \mathrm{hPa}$ isobars on the east coast of Greenland, just north of Cape Farewell. A similar fluctuation was observed at the same location in three other ETJ case studies.

The wind speed peaks at around $450 \mathrm{~km}$, shown by the peak in the Coriolis force. Beyond this point, the wind speed decreases and the jet aligns with the isobars by around $500 \mathrm{~km}$ (i.e. as noted earlier the along-jet pressure gradient is zero here). Past Cape Farewell, the pressuregradient force is due to the difference between the high pressure over Greenland and the low-pressure system passing to the south (Figure 9). There is a region of small imbalance between 500 and $700 \mathrm{~km}$ as the deceleration of the jet causes more turbulent mixing. Beyond $700 \mathrm{~km}$ the cross-jet residual diminishes, the jet straightens as it moves downstream and the flow returns to a state of geostrophic balance.

Given that five of the six terms in Eq. (2) have been plotted, it is clear that the residual of the terms should be equal to the time derivative term, $\partial u / \partial t$, plus any unaccounted-for components of the momentum budget. The $\partial u / \partial t$ term is assumed to be small, as the simulation is approximately steady-state (see Figure 7). To test this assumption, $\partial u / \partial t$ was calculated using a time interval of three hours, and was found to be on average $7.6 \times 10^{-5} \mathrm{~m} \mathrm{~s}^{-2}$, i.e. approximately an order of magnitude smaller that the other terms. Therefore, the non-negligible residual in both cross-jet and alongjet directions warrants further investigation in the next section.

\subsection{Investigating the residual}

Thus far the momentum budget has been calculated using a Cartesian-altitude coordinate system and then rotating the terms into components along and normal to the jet locus. An alternative approach is to convert the equations into natural coordinates. Following the framework of Holton (2004), the Lagrangian derivative, $\mathrm{D} U / \mathrm{D} t$, can be shown to be

$$
\frac{\mathrm{D} \mathbf{U}}{\mathrm{D} t}=\tilde{s} \frac{\mathrm{D} u_{s}}{\mathrm{D} t}+\tilde{n} \frac{\bar{u}_{s}^{2}}{R},
$$

where $u_{s}$ is the along-jet wind speed and $R$ is the radius of curvature. The change to natural coordinates is equivalent to a change to polar coordinates, so it also gives rise to a number of other terms (Batchelor, 1967); however, many of these terms can be ignored since they contain $u_{n}$, the cross-jet wind speed, which is equal to zero, or because they contain a $1 / R^{2}$ factor, which makes them negligible owing to the size of $R$.

Applying this framework to Eq. (2) and removing the negligible terms gives Eq. (4) for the cross-jet momentum budget:

$$
-\underbrace{\frac{\bar{u}_{s}^{2}}{R}}_{\text {centrifugal }}-\underbrace{f \bar{u}_{s}}_{\text {Coriolis }}-\underbrace{\frac{1}{\bar{\rho}} \frac{\partial \bar{p}}{\partial n}}_{\text {pressure }}-\underbrace{\frac{1}{\bar{\rho}}\left[\frac{\partial\left(\overline{\rho u_{n}^{\prime} w^{\prime}}\right)}{\partial z}\right]}_{\text {flux divergence }}=0 .
$$

Both Eqs (4) and (2) will, in practice, not add up to exactly zero when calculated for a jet locus. There will be unaccounted-for components that make up a 'residual'. We will derive this residual by calculating all terms in Eq. (4) explicitly. This requires that $\bar{u}_{s}^{2} / R$ be evaluated. Calculating an appropriate radius is a rather sensitive 


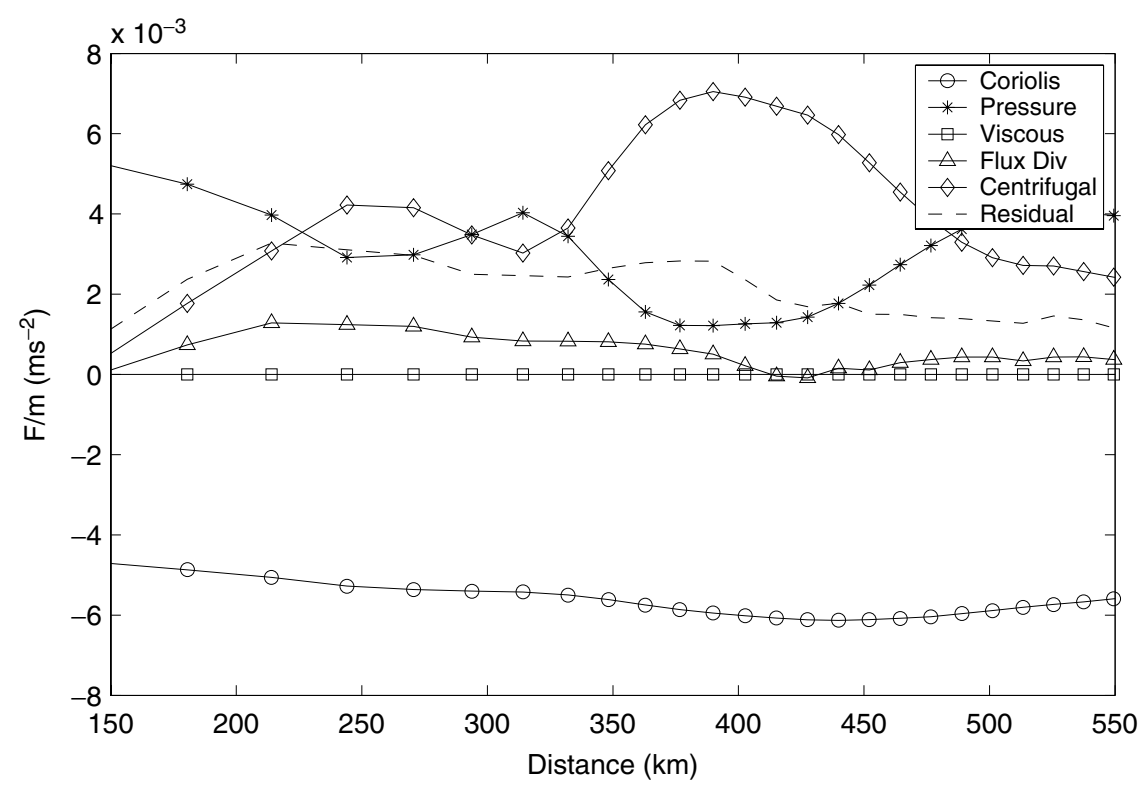

Figure 11. The cross-jet components of the momentum budget shown over the region of imbalance. The forces shown are the Coriolis (circles), pressure gradient (asterisks), viscous stress (squares) and turbluent momentum flux divergence (triangles). The calculated centrifugal force is shown (diamonds) along with the residual (dashed line).

procedure. Here $R$ is calculated by examining the wind direction at two adjacent points on the locus. Since the wind direction at these points is at a tangent to the curve, the difference in wind direction gives the angle of the arc between these two points. Using this angle and the distance between the points, it was possible to calculate the radius. This process was repeated at each point along the locus. The caveat of this approach is that the distance between the two points used was the straight-line distance and not the distance along the arc. This means that the calculated radius will be smaller than the true radius, and hence the calculated centrifugal force will be larger than the true force. However, since the difference between the straight-line distance and the distance along the arc was small compared with the calculated radii, the margin of error in the calculated centrifugal force was found to be less than $2 \times 10^{-6} \mathrm{~m} \mathrm{~s}^{-2}$, or approximately $0.03 \%$.

Figure 11 shows the balance of forces in this natural coordinate framework: note that only the central portion of the jet locus is shown. The centrifugal force is positive and of similar size to the pressure-gradient term over this part of the jet locus. It increases dramatically at around $380 \mathrm{~km}$, at the point of smallest radius. The residual in this plot is now the residual of all five terms of Eq. (4). The approximate inverse correlation between the centrifugal force and the pressure-gradient force reinforces the validity of the approach used to calculate the radius of curvature. Given the size of the residual, it is clear that there is no balance in this region and at this height. Beyond $550 \mathrm{~km}$, the centrifugal force steadily decreases as the curvature in the flow diminishes.

A question remains regarding the relatively large size of the residual terms in these budget calculations, in particular the along-jet residual (Figure 10). As noted earlier, these cannot be explained by changes in time (i.e. the $\partial u / \partial t$ term). Rather these are generated by unaccountedfor contributions to the momentum budget, i.e. differences between Eqs (1), (2) and (4) and the model equations. Examining the momentum budget at various model levels (see Outten (2009) and the next section) shows that these residual terms decrease dramatically with height, becoming negligible in both cross- and along-jet directions by model level $18(1530 \mathrm{~m})$. This implies an approximate gradient wind balance at this level in the jet. Furthermore, as noted earlier, there is a strong correspondence between the residual and the distribution of the turbulent flux divergence term in both cross- and along-jet directions. This suggests that the relatively large residual terms are primarily related to MetUM's ABL scheme (perhaps via model diffusion for example), and may not be common to other dynamical analyses of this case using different numerical models. This question requires further investigation, but is beyond the scope of this study.

From the momentum budgets shown here, the ETJ begins when air decelerates as it approaches the high pressure along the barrier. A cross-jet balance is formed between the pressure-gradient and Coriolis forces. As the jet accelerates off the end of the barrier, the Coriolis force becomes dominant and so anticyclonic curvature is introduced into the jet. This causes an increase in the centrifugal force, and the jet becomes somewhat unbalanced at the height of the jet core. Once the jet has passed Cape Farewell, the wind speeds peak, the flow aligns with the local isobars, and as the centrifugal force decreases and the jet straightens it returns to geostrophic balance.

\section{Sensitivity studies}

After establishing the momentum budget in the previous section, it is important to test for sensitivity to 
changes in height, time and the location of the jet locus. To test for sensitivity to height, the cross-jet momentum budget was calculated on model levels $6,9,15$ and 18. These levels correspond to heights of approximately $150,360,1050$ and $1530 \mathrm{~m}$, respectively. These budgets were calculated for three-hour means centred at 1300 UTC.

The pressure-gradient term was found to be stronger at low levels and to decrease slightly with height (not shown). This was especially noticeable at the beginning of the jet, where at low levels the jet is highly constrained by the orography of Greenland, and it is this pressure gradient that is driving the jet. The distribution of the pressure-gradient force along the jet is very similar at all heights. The advection term also changed slightly with height, associated with the changes in the pressuregradient force. The Coriolis force was found to increase slightly up to model level 12 and decrease above it. This change was associated with the variation in the along-jet wind speed, which shows that the wind speed increases from model level 6 up to model level 12, and then decreases up to model level 18. The turbulent momentum flux divergence was higher at low levels and decreased with height, consistent with a decrease in turbulence away from the surface resulting in a smaller turbulent flux divergence. The residual term decreased (i.e. the flow became more balanced) with increasing height above model level 12. This was especially true for the budget calculated on model level $18(1530 \mathrm{~m})$, as this was still within the jet but above the height of the orography. At this level the ETJ moves from a geostrophic balance to a region of gradient wind balance as it rounds Cape Farewell, before returning to geostrophic balance further downstream. The examination of the forces on these various levels indicated that the overall momentum budget is not particularly sensitive to the height at which it is calculated as long as the calculations are within the jet core and above the surface layer, although the residual is sensitive to height.

In order to check the sensitivity of the momentum budget to changes in time, the budget was calculated on model level 12, three hours earlier and later than the original budget. Small differences were observed in the pressure-gradient term, since the low-pressure system to the south moves eastward during this time. Small changes were also observed in the advection term as it reacted to the changes in pressure gradient. Other than these small differences, the momentum budget does not appear to be sensitive to the time at which it is calculated. This would obviously not be true if it were calculated either as the ETJ was forming or as it dissipated.

The final sensitivity study was to examine the location of the jet locus, something that would vary depending on the time, jet height or meteorological criteria used to pinpoint it. An alternative locus was calculated that was close to the original locus in the vicinity of Cape Farewell, but around $24 \mathrm{~km}$ away from the original locus at either end. The momentum budget was calculated using this new locus on model level 12 at 1300 UTC. The distribution of forces along the jet was, on the whole, very similar to the original budget (Figure 10). The largest difference occurred near the beginning of the jet in the pressure-gradient force (not shown). This is unsurprising, since the new curve was further away from the coast at the northern end, and so it crossed the local isobars at a different angle to the original curve, producing a slightly different pressure gradient. The geostrophic balance at the beginning of the jet was still present when using this alternative new jet locus, but only for the first $40 \mathrm{~km}$. This suggests that the qualitative results of the momentum budget are not sensitive to the location of the jet locus provided it runs approximately along the centre of the jet.

The original momentum budget was calculated at 1300 UTC, approximately the central time of the ETJ's lifetime, at $660 \mathrm{~m}$, approximately the central height for most of the jet, using a curve fitted through the core of the jet as defined by the maxima in wind speed. Sensitivity studies indicate that using an alternative height, time or jet locus does not significantly change the fundamental balance of forces in this ETJ. Further details can be found in Outten (2009).

\section{Additional easterly tip jet case studies}

Following the momentum budget analysis of the 21 February 2007 case, an obvious next step was to see whether this force balance is similar in other ETJ cases. Three more cases were selected for investigation: 13 January 2008, 11 January 2008 and 10 February 2007. The synoptic situation in each case was fairly similar: an easterly flow, a passing low-pressure system to the south of Cape Farewell, a strong pressure gradient along the southeast coast of Greenland, etc. QuikSCAT observations showed a high-wind-speed jet curving anticyclonically around the Cape in each case (Outten, 2009).

Figure 12 shows the cross-jet momentum budget for these three cases, each calculated along a locus appropriate to the individual jet and oriented as on a pseudostreamline. In all three budgets the residual is low at the beginning, indicating a balance between the Coriolis force and the cross-jet component of the pressure-gradient force, and at the end of the jet, where the flow is in approximate geostrophic balance. The pressure-gradient force decreases as the jet rounds the Cape and increases as the jet moves downstream. The rise in the residual highlights the unbalanced region where the centrifugal force rises and falls as the jet curves and straightens (see Figure 11). Note that in each case the pressure-gradient force also shows distinctive oscillation as the jet passes the two large fjords, as discussed earlier (as seen in Figure 10 for the 21 February case). Overall, the momentum budget for all three cases is similar to the original momentum budget for the 21 February case shown in Figure 10. This suggests that the momentum budget analysis is not dependent on this case, but appears to be more generally applicable. 

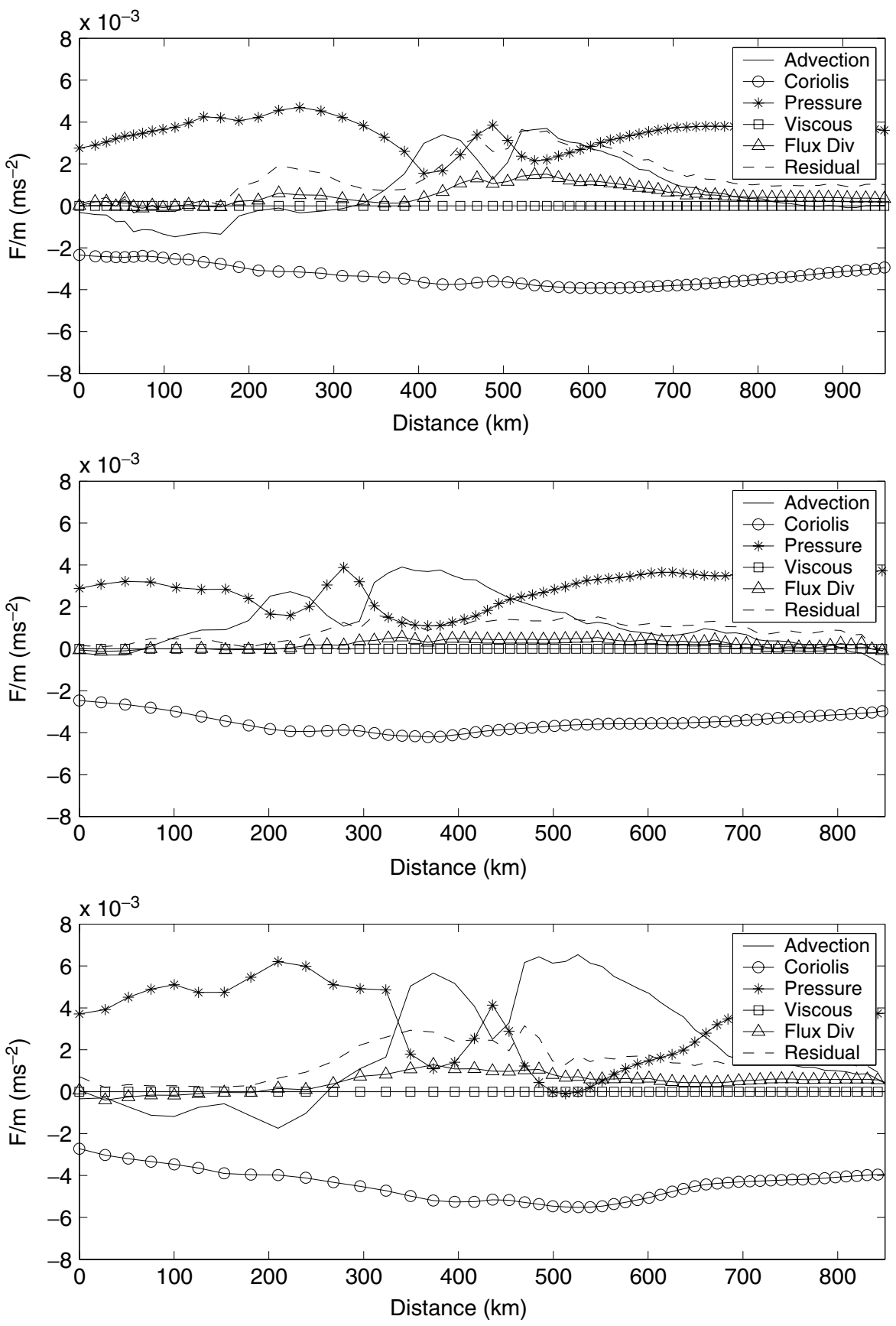

Figure 12. The cross-jet components of the momentum budget for 13 January 2008 (top), 11 January 2008 (middle) and 10 February 2007 (bottom) are shown against distance along the jet locus. The forces shown in each plot are the advection (solid line), Coriolis (circles), pressuregradient (asterisks), viscous stress (squares) and turbulent momentum flux divergence (triangles). The residual is also shown (dashed lines).

\section{Conclusions}

This article continues the investigation of the easterly tip jet observed on 21 February 2007 during the GFDex campaign. Part I of this investigation discussed the observations taken during the field campaign (Renfrew et al., 2009a). Part II of the investigation has examined numerical simulations of this event using the MetUM version 6.1 and has used those simulations to derive a momentum budget for the easterly tip jet. This budget was shown to be robust and to be generally applicable for other ETJ cases.
Comparisons to dropsondes suggested that the wind speeds in the northern end of the jet were too low in the default model simulation, and that the model's boundary layer temperature was too cold by $3-4 \mathrm{~K}$. These problems were resolved by improving the roughness lengths used in the parametrization scheme to be more in line with current research, and by replacing the sea-ice and seasurface temperature fields used in the model with fields from the OSTIA dataset.

The model simulations indicate that as the flow approaches the region of high pressure along the coast of southeast Greenland, the air decelerates and turns, 

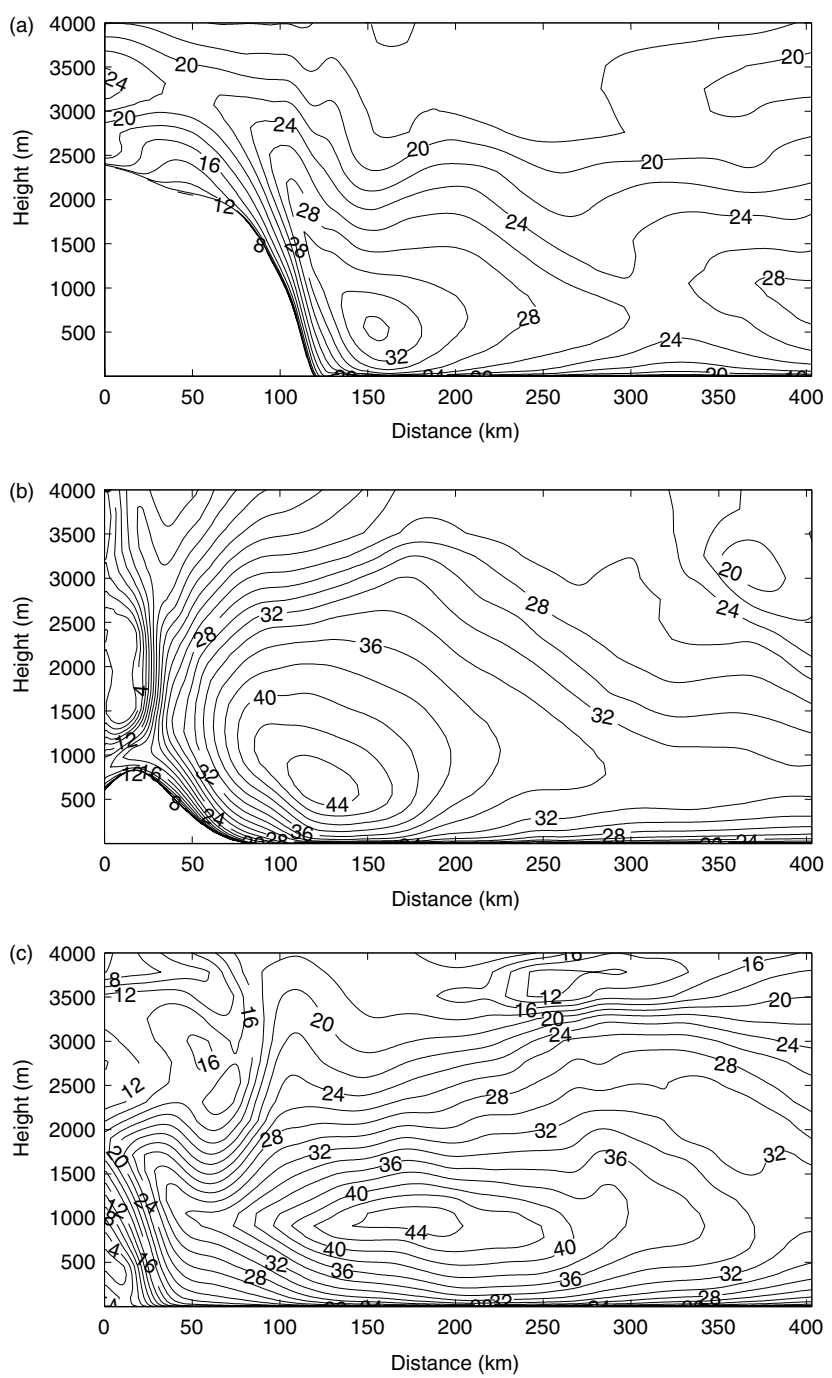

Figure 13. Wind speed with contours every $2 \mathrm{~m} \mathrm{~s}^{-1}$ in cross-sections through the jet, located at the first, second and fourth points where variability in the jet was examined (i.e. the grey squares in Figure 8) and approximately perpendicular to the jet locus.

becoming more cross-isobaric. This is similar to what is seen in simulations of the 'left-sided jets' investigated by Barstad and Grønås (2005).

An analysis of the momentum budget was derived that showed three distinct dynamical regions along the ETJ. Figure 13 highlights these regions by showing wind speed in three cross-sections through the jet. At the beginning, the cross-jet forces are balanced as in a barrier flow (Figure 13(a)). However, the along-jet component of the pressure-gradient force accelerates the jet parallel to the orography. As the jet approaches Cape Farewell, the constraining orography decreases in height, the pressure-gradient force decreases and the flow enters a second region where it becomes unbalanced at the jet core height (Figure 13(b)). In this region, the Coriolis force is dominant and a characteristic anticyclonic curvature is introduced into the jet. This gives rise to a centrifugal force, and is accompanied by an increase in the turbulent momentum flux divergence as illustrated in Figure 11. At higher levels in this region the residual decreases, leading to an approximate gradient wind balance at around $1500 \mathrm{~m}$, which is above the height of the orography. Moving further downstream away from the orography, the jet moves into its third region and begins to settle out into the surroundings (Figure 13(c)). Here, the jet aligns with the local isobars and becomes shallower, the centrifugal force decreases and the jet slows and straightens, returning to geostrophic balance, before finally dissipating into the prevailing synoptic situation.

The momentum budget analysis has been subjected to a series of sensitivity tests for different locus heights, times and locations. In all of these tests, small differences were observed but the general balance of forces remained the same, assuming that the budget is calculated in the jet core while it is in steady state (i.e not during its formation or dissipation) and that the jet locus used is approximately central to the core of high winds.

The momentum budgets of three other ETJs were also examined. In each of these cases, broadly the same distribution of forces was found along the length of the jet, suggesting that the underlying dynamics found here for the 21 February 2007 case also holds for other easterly tip jets off Cape Farewell. Specifically, the ETJ goes through three stages as it rounds Cape Farewell: cross-jet geostrophic balance, an unbalanced stage at the core level - which is close to gradient wind balance higher up in the jet - and then a return to geostrophic balance downstream.

\section{Acknowledgements}

The authors thank Sue Gray for useful discussions, the Met Office for making the MetUM available and NCAS for technical support with the MetUM. Discussions with Ariadne Outten and Hanneke Luijting were also greatly appreciated. Three anonymous reviewers are thanked for comprehensive reviews which have improved this article. This work was funded by the Natural Environmental Research Council (Grant NE/C003365/1).

\section{References}

Andreas EL, Persson POG, Jordan RE, Horst TW, Guest P, Grachev AA, Fairall CW. 2005. Parameterizing the turbulent surface fluxes over summer sea ice. In Eighth Conference on Polar Meteorology and Oceanography. Am. Meteorol. Soc.: San Diego, CA.

Barstad I, Grønås S. 2005. Southwesterly flows over southern Norway - mesoscale sensitivity to large-scale wind direction and speed. Tellus A 57: 136-152.

Batchelor GK. 1967. An Introduction to Fluid Dynamics. Cambridge University Press: Cambridge, UK.

Chelton DB, Freilich . 2005. Scatterometer-based assessment of 10-m wind analyses from the operational ECMWF and NCEP numerical weather prediction models. Mon. Weather Rev. 133: 409-429.

Davies T, Cullen M, Malcolm A, Mawson M, Staniforth A, White A, Wood N. 2005. A new dynamical core for the Met Office's global and regional modelling of the atmosphere. Q. J. R. Meteorol. Soc. 131: $1759-1782$.

Donlon C, Robinson I, Casey KS, Vazquez-Cuervo J, Armstrong E, Arino O, Gentemann C, May D, LeBorgne P, Piolle J, Barton I, Beggs H, Poulter DJS, Merchant CJ, Bingham A, Heinz S, Harris A, Wick G, Emery B, Minnett P, Evans R, Llewellyn-Jones D, Mutlow C, Reynolds RW, Kawamura H, Rayner N. 2007. The global 
ocean data assimilation experiment high-resolution sea surface temperature pilot project. Bull. Am. Meteorol. Soc. 88: 1197-1213.

Doyle JD, Shapiro MA. 1999. Flow response to large-scale topography: the Greenland tip jet. Tellus A 51: 728-748.

Heinemann G, Klein T. 2002. Modelling and observations of the katabatic flow dynamics over Greenland. Tellus A 54: 542-554.

Holton JR. 2004. An Introduction to Dynamic Meteorology. Academic Press: New York.

Kristjánsson JE, McInnes H. 1999. The impact of Greenland on cyclone evolution in the North Atlantic. Q. J. R. Meteorol. Soc. 125: 2819-2834.

Lock AP, Brown AR, Bush MR, Martin GM, Smith RNB. 2000. A new boundary layer mixing scheme. Part I: Scheme description and single-column model tests. Mon. Weather Rev. 128: 3187-3199.

Martin R, Moore GWK. 2007. Air-sea interaction associated with a Greenland reverse tip jet. Geophys. Res. Lett. 34: L24802. DOI: $10.1029 / 2007$ GL031093.

Moore GWK, Renfrew IA. 2005. Tip jets and barrier winds: A QuikSCAT climatology of high wind speed events around Greenland. J. Climate 18: 3713-3725.

Moore GWK, Pickart RS, Renfrew IA. 2008. Buoy observations from the windiest location in the world ocean, Cape Farewell, Greenland. Geophys. Res. Lett. 35: L18802. DOI:10.1029/2008GL034845.

Outten S. 2009. Easterly tip jets around Greenland. PhD Thesis. Univ. East Anglia: UK.

Parish TR. 1983. The influence of the Antarctic Peninsula on the wind field over the Western Weddell Sea. J. Geophys. Res. 88: 2684-2692.

Petersen GN, Renfrew IA. 2009. Aircraft-based observations of air-sea fluxes over Denmark Strait and the Irminger Sea during high wind speed conditions. Q. J. R. Meteorol. Soc. 135: 2030-2045.

Petersen GN, Ólafsson H, Kristjánsson JE. 2003. Flow in the lee of idealized mountains and Greenland. J. Atmos. Sci. 60: 2183-2195.

Petersen GN, Kristjánsson JE, Ólafsson H. 2005. The effect of upstream wind direction on atmospheric flow in the vicinity of a large mountain. Q. J. R. Meteorol. Soc. 131: 1113-1128.

Petersen GN, Renfrew IA, Moore GWK. 2009. An overview of barrier winds off Southeastern Greenland during GFDex. Q. J. R. Meteorol. Soc. 135: 1950-1967.
Renfrew IA. 2004. The dynamics of idealized katabatic flow over a moderate slope and ice shelf. Q. J. R. Meteorol. Soc. 130: 1023-1045. DOI:10.1256/qj.03.24.

Renfrew IA, Moore GWK, Kristjánsson JE, Ólafsson H, Gray SL, Petersen GN, Bovis K, Brown P, Fore I, Haine T, Hay C, Irvine EA, Oghuishi T, Outten S, Pickart R, Shapiro MA, Sproson D, Swinbank R, Wooley A, Zhang S. 2008. The Greenland Flow Distortion experiment. Bull. Am. Meteorol. Soc. 88: 1307-1324.

Renfrew IA, Outten SD, Moore GWK. 2009a. An easterly tip jet off Cape Farewell, Greenland. Part I: Aircraft observations. $O . J$. R. Meteorol. Soc. 135: 1919-1933.

Renfrew IA, Petersen GN, Moore GWK, Sproson DAJ, Adiwidjaja H, Zhang S, North R. 2009b. A comparison of aircraft-based surfacelayer observations over Denmark Strait and the Irminger Sea with meteorological analyses and QuikSCAT winds. Q. J. R. Meteorol. Soc. 135: 2046-2066.

Schwerdtfeger W. 1975. Effect of Antarctic Peninsula on the temperature regime of the Weddel Sea. Mon. Weather Rev. 114: 45-51.

Smith RB. 1982. Synoptic observations and theory of orographically disturbed wind and pressure. J. Atmos. Sci. 39: 60-70.

Sproson DAJ, Renfrew IA, Heywood KJ. 2008. Atmospheric conditions associated with oceanic convection in the south-east Labrador Sea. Geophys. Res. Lett. 35: L06601. DOI:10.1029/2007GL032971.

Staniforth A, White A, Wood A, Thuburn J, Zerroukat M, Cordero E, Davies T. 2003. Joy of U.M. 5.5 - Model formulation. Unified Model Documentation 15.

Stark J, Donlon C, Martin M, McCulloch M. 2007. OSTIA: An operational, high resolution, real time, global sea surface temperature analysis system. OCEANS 2007 - EUROPE 1-3: 331-334.

Stull RB. 1988. An Introduction to Boundary Layer Meteorology. Springer: Berlin.

Wentz FJ. 1997. A well-calibrated ocean algorithm for SSM/I. J. Geophys. Res. 102: 8703-8718.

Zhong S, Whiteman C. 2008. Downslope flows on a low-angle slope and their interactions with valley inversions. Part II: numerical modelling. J. Appl. Meteorol. Clim. 47: 2039-2057. 